\title{
The Application of Improved Position for Lower Limb full Length DR Radiograph in Hip/Knee Arthroplasty
}

\author{
Huige Hou ${ }^{1}$, Jinguo Lai ${ }^{2}$, Xiufang Yan $^{2}$, Zhichao Lin ${ }^{2, *}$ \\ ${ }^{1}$ Department of Orthopedics, the First Affiliated Hospital of Jinan University, Guangzhou, China \\ ${ }^{2}$ Medical Imaging Center, the First Affiliated Hospital of Jinan University, Guangzhou, China \\ Email address: \\ 18620085802@126.com (Zhichao Lin) \\ ${ }^{*}$ Corresponding author
}

To cite this article:

Huige Hou, Jinguo Lai, Xiufang Yan, Zhichao Lin. The Application of Improved Position for Lower Limb full Length DR Radiograph in Hip/Knee Arthroplasty. Journal of Surgery. Vol. 6, No. 2, 2018, pp. 33-35. doi: 10.11648/j.js.20180602.11

Received: January 16, 2018; Accepted: January 31, 2018; Published: March 15, 2018

\begin{abstract}
Aim Compared with the traditional position, we tried to explore the application of improved position for lower limb DR radiograph in hip and knee arthroplasty. Method Retrospective analysis of the traditional and improved DR radiographs from 60 cases for hip and knee arthroplasty, by comparing the division angle, lower limb mechanical axis length, mechanical deviation between the lower limb mechanical axis and the center of the tibial plateau. Results (1) Preoperative data from twenty cases of improved and 15 cases of traditional lower limb DR radiographs showed the mechanical deviations between the lower limb mechanical axis and the center of the tibial plateau were $28.18,4.71,30.66,11.92$; postoperative data from fifty cases of improved and 33 cases of traditional DR radiographs showed the mechanical deviations between the lower limb mechanical axis and the center of the tibial plateau were 5.04, $11.03(\mathrm{P}<0.05)$. (2) Improved position much more clearly showed the full length of the lower limb, compared with traditional position. (3) Values preoperative correction angle of 31 cases of improved and 19 cases of traditional position were $5.9^{\circ}, 6.7^{\circ}(\mathrm{P}<0.05)$. Conclusion $\mathrm{DR}$ radiographs of improved position can realistically show the preand post-operative status of hip and knee. The data of correction angle, lower limb mechanical axis length, mechanical deviations between the lower limb mechanical axis and the center of the tibial plateau can be more accurate. It is more convenient for the selection of operation procedure and for the assessment of surgery and rehabilitation.
\end{abstract}

Keywords: Arthroplasty, DR Radiograph, Lower Limb Full Length, Traditional Body Position, Improved Body Position

\section{Introduction}

As the demand of better quality of life, arthroplasty becomes more and more eye-catching by people with hip and knee problems. The aim of arthroplasty is to relieve pain and to improve the function of joints. The main disadvantage of arthroplasty is the unequal length of the lower limbs. According to the literature, if the unequal length of lower limbs is more than $2.5 \mathrm{~cm}$, sciatic nerve symptoms will appear, which would severely impact the postoperative satisfaction and patients' living quality. Full-length lower limb radiographs can clearly show the situation of both lower limbs, providing important clinical data for the pre- and post-operative judgement of arthroplasty. Whether or not the body position for DR photograph is standard would directly affect the quality of calculation. The current study retrospectively analyzed the pre- and post-operative DR radiograph data of 60 patients under total hip or knee arthroplasty. The values of correction angle, lower limb mechanical axis length, mechanical deviations between the lower limb mechanical axis and the center of the tibial plateau were determined. The application value of improve body position for DR radiograph in arthroplasty was explored.

\section{Clinical Data and Methods}

\subsection{Data Collection}

Retrospective analysis was performed on sixty cases under arthroplasty from Jan 2013 to Jan 2015 in the First Affiliated Hospital of Jinan University. Inclusion criteria: (1) All patients were taken pre- and post-operative DR radiographs of lower limbs under the DR machine (GE corporation), with the same 
position and film scale; (2) for the first arthroplasty; (3) with complete pre- and post-operative data, with better follow-up for 6 months. Meeting the criteria, there were: 13 cases of knee arthroplasty and 47 cases of hip arthroplasty; 35 cases of male and 25 of female; 34 cases of equal shoulder standing position and 26 cases of none.

\subsection{Photography Method and Body Position}

Place the detector vertically, make the tube $180 \mathrm{~cm}$ away from the detector, place the camera away $1-2 \mathrm{~cm}$ from the detector, to make the synchronous movement of all these devices. Traditional unequal shoulder standing position: standing on the photography shelf, with back close to the shelf, hands naturally drooping, feet close together, showing anatomical body gesture. The improved equal shoulder position: front and rear position, patella forward, foot show equal width of shoulder, the internal rotation of about $15^{\circ}$.

\subsection{Photography Processing and Measurement}

After the completion of image stitching, the DR images were uploaded for analysis and measurement for the following parameters: the correction angle, lower limb mechanical axis length, mechanical deviation between the lower limb mechanical axis and the center of the tibial plateau. Lower limb full length: from the top of the femoral head to the center of the ankle joint [1]; lower limb mechanical axis length: from midpoint of the femoral joint to the midpoint of the medial ankle joint; the correction angle: the angle of the femoral mechanical axis and the femoral axis.

\subsection{General Evaluation}

The normal reference value of correction angel of Chinese: male, $5.6 \pm 0.9^{\circ}$, female, $5.7 \pm 1.0^{\circ}$. The mechanical deviation between the lower limb mechanical axis and the center of the tibial plateau: less than $5 \mathrm{~mm}$ defines excellent; $5-10 \mathrm{~mm}$ defines normal. The data were analyzed by SPSS 20.0, paired t-test for statistical analysis, $\mathrm{p}<0.05$ showed significant differences.

\section{Results}

Patients were divided into two groups: (1) improved position group and (2) traditional position group. The data showed that improved position significantly decreased the mechanical deviation between the lower limb mechanical axis and the center of the tibial plateau, making the score value approaching excellent $(p<0.05$, Table 1$)$. The lower limb full-length between the two groups showed no significant difference $(\mathrm{p}>0.05$, Table 2$)$. The preoperative correction angle showed significant difference $(\mathrm{p}<0.05$, Table 3$)$. The pre- and post- operative mechanical deviation between the lower limb mechanical axis and the center of the tibial plateau in improved group decreased significantly than that in the traditional control group ( $\mathrm{p}<0.05$, Table 4 and 5).

Table 1. The comparison of improved and traditional post-operative mechanical deviation between the lower limb mechanical axis and the center of the tibial plateau.

\begin{tabular}{|c|c|c|c|c|}
\hline Deviation difference & Cases & Mean & SD & SE \\
\hline 1 Improved position & 50 & 5.0460 & 3.90971 & 0.55292 \\
\hline \multirow[t]{2}{*}{2 Traditional position } & 33 & 11.9303 & 7.61460 & 1.32553 \\
\hline & $\mathrm{t}$ & df & $\mathrm{p}$ & \\
\hline Assuming equal variance & -5.413 & 81 & 0.0001 & \\
\hline Assuming unequal variance & -4.793 & 43 & 0.0001 & \\
\hline
\end{tabular}

Table 2. The comparison of post-operative lower limb full-length.

\begin{tabular}{lllll}
\hline Lower limb full-length & Cases & Mean & SD & SE \\
\hline 1 Improved position & 37 & 4.7595 & 3.26245 \\
2 Traditional position & 23 & 4.1478 & 4.15603 \\
& $\mathrm{t}$ & $\mathrm{df}$ & $\mathrm{p}$ & 0.53634 \\
& -0.403 & 58 & 0.6881 & 0.86659 \\
Assuming equal variance & -0.381 & 38.619 & 0.705 \\
\hline
\end{tabular}

Table 3. The pre-operative correction angle analysis between the two groups.

\begin{tabular}{lllll}
\hline Correction angle & Cases & Mean & SD & SE \\
\hline 1 Improved position & 31 & 5.9097 & 1.43535 & 0.25780 \\
2 Traditional position & 19 & 6.79478 & 1.32559 & 0.30411 \\
& $\mathrm{t}$ & $\mathrm{df}$ & 48 & 0.034 \\
Assuming equal variance & -2.177 & 40.589 & 0.032 \\
Assuming unequal variance & -2.220 & & \\
\hline
\end{tabular}

Table 4. The improved position group: pre-and post-operative mechanical deviation between the lower limb mechanical axis and the center of the tibial plateau.

\begin{tabular}{llllll}
\hline Improved position & Cases & Mean & SD & SE & t \\
\hline 1 preoperative & 20 & 28.185 & 16.123 & 3.605 & \\
2 postoperative & 20 & 4.710 & 3.0071 & 0.672 & 3.835 \\
3 preoperative - postoperative & 20 & 23.475 & 17.151 & 0.000 & 6.121 \\
\hline
\end{tabular}


Table 5. The traditional position group: pre- and post-operative mechanical deviation between the lower limb mechanical axis and the center of the tibial plateau.

\begin{tabular}{llllll}
\hline Traditional position & Cases & Mean & SD & SE & t \\
\hline 1 preoperative & 15 & 30.660 & 16.117 & 4.161 & 1.629 \\
2 postoperative & 15 & 11.920 & 6.309 & 18.275 & 4.718 \\
3 preoperative - postoperative & 15 & 18.740 & 0.001 & 3.971 \\
\hline
\end{tabular}

\section{Discussion}

Hip or knee arthroplasty is the most effective approach for the treatment of late osteoarthritis, rheumatoid arthritis, femoral head necrosis and other knee/hip diseases. Despite of the rapid development of the technique over the sixty years, there appears many complications, such as unequaled lower limb length, prosthetic loosening, prosthetic infection, fracture and dislocation around the prosthesis [2-4]. These complications greatly affect the surgery satisfaction and the living quality of patients. DR images can clearly show the situation of both legs, suppling important clinical information for surgeries [5]. As the critical step for arthroplasty, imaging measurement plays an important role for preoperative assessment, accurate intraoperative osteotomy and osteotomy angel determination and for postoperative quality evaluation [6]. Also, DR images are the most reliable and direct method to measure the leg length.

Currently, mechanically dissecting position is the way mostly used to take DR photograph, which is also called the unequal shoulder standing position. In the criteria of this research, patella of most patients is not correct and the overlap of tibia and fibular head is over one third, which are greatly affect the preoperative assessment. Thus, the improved position is applied. Many orthopedic surgeons believe that improved position can truly reflect the lower limb force line, especially for patients whose joints were badly damaged. When traditional standing position cannot be applied, the normal angle of lower limb mechanical axis passes the center of the tibial plateau is around $57.8 \%[6,7]$, which is one of the important standard value to assess arthroplasty surgery. Another important standard value is the mechanical deviation between the lower limb mechanical axis and the center of the tibial plateau, of which less than $5 \mathrm{~mm}$ defines excellent and 5-10 mm defines normal [8-10]. Using different position for DR images results greatly different measured data, and our results show that the application of improved position is more suited for arthroplasty surgeries. Improved position for DR photograph can be more accurate to reflect the situation of the joint pre- or post- operatively, is more benefit for the evaluation of the success of arthroplasty surgeries.

In summary, calculations from DR images of improved position can reflect the real clinical measurement, and reflect the actual situation of pre- and post- operative knee/ hip arthroplasty, compared with traditional position DR images. The data from improved position can be more accurate in mechanical deviation between the lower limb mechanical axis and the center of the tibial plateau and correction angle analysis. In general, if use improved position for DR photograph, the rate of acquiring equal leg length can be largely increased, supply critical information for prognosis and increase surgery satisfaction and the quality of patient's living life.

\section{Acknowledgements}

The authors declare no conflict of interest.

\section{References}

[1] M. B. Siqueira, D. Ramanathan, A. K. Klika, C. A. Higuera, W. K. Barsoum, Role of negative pressure wound therapy in total hip and knee arthroplasty, World J Orthop, 2016, (7): 30-37.

[2] S. R. Piedade, A. Pinaroli, E. Servien, P. Neyret, Revision after early aseptic failures in primary total knee arthroplasty, Knee Surg Sports Traumatol Arthrosc, 2009, (17): 248-253.

[3] T. K. Fehring, S. Odum, W. L. Griffin, J. B. Mason, M. Nadaud, Early failures in total knee arthroplasty, Clin Orthop Relat Res, 2001, DOI: 315-318.

[4] T. O. White, T. W. Dougall, Arthroplasty of the hip. Leg length is not important, J Bone Joint Surg Br, 2002, (84): 335-338.

[5] S. C. Tipton, J. K. Sutherland, R. Schwarzkopf, The Assessment of Limb Length Discrepancy Before Total Hip Arthroplasty, J Arthroplasty, 2016, (31): 888-892.

[6] G. Meermans, A. Malik, J. Witt, F. Haddad, Preoperative radiographic assessment of limb-length discrepancy in total hip arthroplasty, Clin Orthop Relat Res, 2011, (469): 1677-1682.

[7] A. Konyves, G. C. Bannister, The importance of leg length discrepancy after total hip arthroplasty, J Bone Joint Surg Br, 2005, (87): 155-157.

[8] Y. Zhang, W. He, T. Cheng, X. Zhang, Total Hip Arthroplasty: Leg Length Discrepancy Affects Functional Outcomes and Patient's Gait, Cell Biochem Biophys, 2015, (72): 215-219.

[9] M. R. Whitehouse, N. S. Stefanovich-Lawbuary, L. R. Brunton, A. W. Blom, The impact of leg length discrepancy on patient satisfaction and functional outcome following total hip arthroplasty, J Arthroplasty, 2013, (28): 1408-1414.

[10] S. P. Krishnan, R. W. Carrington, S. Mohiyaddin, N. Garlick, Common misconceptions of normal hip joint relations on pelvic radiographs, J Arthroplasty, 2006, (21): 409-412. 